\title{
MODELO BRASILEIRO DE MATURIDADE PARA CIDADES INTELIGENTES: ANÁLISE DOS MUNICÍPIOS DO ESTADO DE SANTA CATARINA
}

\author{
Darlan Junckes \\ Clarissa Stefani Teixeira \\ darlanjunckes@gmail.com \\ clastefani@gmail.com \\ Universidade Federal de Santa Catarina - UFSC
}

Resumo: Nunca antes tantas pessoas viveram em aglomerados urbanos e este fato requer que as cidades estejam preparadas para atender as demandas de serviços e de infraestrutura. Neste sentido, o objetivo do artigo é analisar o desempenho de seis cidades do estado de Santa Catarina com melhores índices de desenvolvimento sustentável, através do Modelo Brasileiro de Maturidade para Cidades Inteligentes, com dados obtidos por meio do Sistema de Indicadores de Desenvolvimento Municipal Sustentável (SIDMS), promovido pela Federação Catarinense de Municípios (FECAM). Foram calculadas médias dos índices dos municípios para os domínios de educação, saúde e água, além da identificação dos indicadores que influenciaram nos resultados.

Palavras chave: Sustentabilidade, Modelo de Maturidade, Cidades Inteligentes, SmartCities, SIDMS

Abstract: Never before have so many people lived in urbana reas and this fact requires that cities are prepared to meet the demands of services and infrastructure. In this sense, the objective of this articleis to analyze the performance of six cities in the state of Santa Catarina with better indices of sustain able development through the Brazilian Smart Cities Maturity Model, with data obtained through the Sustainable Municipal Development Indicators System (SIDMS), sponsored by the Santa Catarina Federation of Municipalities (FECAM). Averages were calculated indexes of municipalities to areas of education, health and water, in addition to identifying indicators that influenced the results.

Keywords: Sustainability, MaturityModel, Smart Cities, SIDMS.

\section{Introdução}

Em 2014, 3,88 bilhões de pessoas residiam em áreas urbanas, conforme aponta o estudo World Urbanizations Prospects, realizado pela Organização das Nações Unidas (WUP ONU, 2014). A estimativa é que até $2050,66 \%$ da população mundial resida em cidades, sendo que no Brasil este índice deve alcançar a marca de 91\% (ONU, 2014). As próximas décadas representarão profundas mudanças no perfil das cidades ao redor do mundo, uma vez que suas densidades demográficas apresentam comportamento crescente.

Apesar de concentrarem a maior parte da população, as cidades ocupam apenas 2\% da área total do planeta e consomem 75\% dos recursos utilizados (BOUSKELA, et al., 2016). Os ambientes urbanos possuem inúmeros desafios que envolvem diferentes atores unidos em um 
sistema de alta complexidade. Para o atendimento das necessidades dos cidadãos, os gestores das cidades devem fomentar a inovação em áreas específicas a fim de promover o crescimento sob o tripé da sustentabilidade (HOLLANDS, 2008).

O conceito de Cidade Inteligente ganhou ênfase nos últimos anos, sendo inicialmente considerada como um ambiente capaz de fazer a conexão entre o digital e o real, delimitado em uma área geográfica com alta concentração de conhecimento, tendo sua gestão pautada em tecnologias de informação e comunicação (KOMNINOS, 2002). O autor diz ainda que cidades inteligentes são áreas com grande capacidade inovativa, constituídas através da produção intelectual das suas populações, bem como o emprego da gestão de conhecimento e da comunicação como forma de melhoria da estrutura e dos serviços na cidade, aumentado assim a qualidade de vida das pessoas.

É sabido que uma cidade torna-se inteligente não apenas pelo emprego de tecnologias, mas também pela forma inteligente de sua administração, orientada pela eficiência da gestão. Neste sentido, umas martcitie devem ter como premissa a promoção da qualidade de vida de sua população, seja pelo desenvolvimento econômico, com a geração de empregos e renda; ou como por meio da oferta de estrutura básica em saúde, saneamento, água, educação e preservação do meio ambiente (RAMOS JUNIOR; GALIOTTO, 2013). Isso assegura que futuras gerações poderão usufruir de cidades com altos índices de desenvolvimento econômico, social e ambiental.

Desta forma, o estudo objetiva a mensuração da capacidade de desenvolvimento dos municípios catarinenses com iniciativas de cidades inteligentes, tendo como base o Modelo Brasileiro de Maturidade para Cidades Inteligentes.

\section{Os indicadores de Cidades Inteligentes}

Algumas iniciativas são encontradas no mundo para considerar uma Cidade Inteligente. Dentre os índices mais divulgados, pode ser citado o EuropeanSmartCities ${ }^{1}$. Na Europa, por meio de um trabalho colaborativo entre o Centro de Ciência Regional da Universidade de Tecnologia de Vienna, o Departamento de Geografia da Universidade de Ljubljana, e o Instituto de Pesquisa para Habitação, Urbanismo e Estudos de Mobilidade da Universidade de Tecnologia Delft, foi desenvolvida uma metodologia para verificar a performance das cidades. Para descrever uma cidade inteligente são seis características (economia inteligente, pessoas inteligentes, governança inteligente, mobilidade inteligente, meio ambiente inteligente, vida inteligente), 31 fatores e 74 indicadores (EUROPEAN SMART CITIES, 2016).

No Brasil, a Urban Systems, empresa que oferece soluções estratégicas e competitivas que apóiam o processo decisório e o planejamento de projetos de base imobiliária, em parceria com a Sator, empresa organizadora do evento homônimo, apresentam o Ranking ConnectedSmartCities (CONNECTED SMART CITIES, 2016). Este considera o desenvolvimento de 11 eixos, os quais são: Mobilidade, Urbanismo, Meio Ambiente, Energia, Tecnologia e Inovação, Economia, Educação, Saúde, Segurança, Empreendedorismo e Governança.

Outras iniciativas vêm sendo consideradas, como por exemplo, IeseCities in Motion (IESE BUSINESS SCHOOL; UNIVERSITY OF NAVARRA, 2014). No Brasil, o estudo de Afonso et al. (2003) apresenta além dos indicadores um índice de maturidade para cidades inteligentes.

\section{O Modelo Brasileiro de Maturidade para Cidades Inteligentes}

\footnotetext{
${ }^{1}$ EuropeanSmartCities. Disponível em: < $\underline{\text { http://www.smart-cities.eu/>. }}$
} 
O Modelo Brasileiro de Maturidade para cidades Inteligentes está sendo elaborado mediante a disponibilidade de dados em domínios públicos sobre os municípios brasileiros, bem como a capacidade de comparação destes com levantamentos realizados em outras cidades do mundo (AFONSO et al., 2013). O padrão proposto leva em consideração dez domínios, sendo estes: Educação, Saúde, Água, Energia, Governança, Segurança, Meio Ambiente, Habitação, Tecnologia e Transporte. Porém, tratando-se de um modelo ainda em desenvolvimento, sua primeira versão aborda os três domínios considerados mais básicos: Educação, Saúde e Água.

A educação tem a capacidade de influenciar, de forma direta e indireta, vários outros domínios de uma cidade, considerando a proporcionalidade entre o aumento nos níveis de desenvolvimento e os investimentos na área (AFONSO et al., 2013; NEGRI; LEMOS, 2009). Conforme Malek (2009) a educação é um domínio indispensável na definição de cidades inteligentes, sendo observada sua presença em diversos modelos de maturidade.

A Saúde é considerada uma condição fundamental para a promoção da qualidade de vida, sendo entendida como o equilíbrio entre o bem-estar físico, emocional e social, conforme proposta apresentada na Primeira Conferência Internacional sobre Promoção da Saúde, em 1986 (citado por SOUZA; CARVALHO, 2003).

A Água é considerada um domínio essencial para o desenvolvimento de ambientes urbanos, levando em consideração que sua gestão está atrelada a resultados na qualidade de vida da população bem como no crescimento da competitividade de um ambiente, sob diversos aspectos (DEAKIN; ALLWINKLE, 2007; AFONSO et al., 2013).

Para a mensuração da maturidade de uma cidade inteligente, o Br-SCMM elenca cinco níveis de discussão, visando responder questões que levem as cidades a promover evolução nos domínios que possuírem maior capacidade (AFONSO et al., 2013): O Quadro 1 ilustra o nível e sua descrição.

Quadro 1: Níveis de mensuração da capacidade de transformação em Cidade Inteligente.

\begin{tabular}{|l|l|}
\hline Nível & Descrição \\
\hline Simplificado & $\begin{array}{l}\text { Mensuração de índices para domínios de Educação, Saúde e } \\
\text { Água. }\end{array}$ \\
\hline Gerenciado & Identificação das ações que resultaram nestes índices \\
\hline Aplicado & $\begin{array}{l}\text { Aplicação de novas políticas com a finalidade de alcançar } \\
\text { melhores resultados }\end{array}$ \\
\hline Medido & $\begin{array}{l}\text { Estabelecimento de novos levantamentos de desempenho após as } \\
\text { ações de melhoria }\end{array}$ \\
\hline Transformado & Verificação dos resultados alcançados. \\
\hline
\end{tabular}

Segundo a metodologia utilizada pelos autores, a aplicação do Modelo de Maturidadepara Cidades Inteligentes é um processo sequencial, onde, primeiramente, é realizado levantamento de dados para os domínios abordados, para medir o quanto uma cidade pode se tornar inteligente. $\mathrm{Na}$ sequência buscam-se identificar quais são as práticas adotadas por cada cidade que resultaram nos índices previamente levantados. Após isso, o Br-SCMM propõe a aplicação de novas políticas públicas com a finalidade de fomentar o desenvolvimento nas áreas com piores resultados e, ao fim do processo, realizar novos levantamentos de índices, de forma a comparar a evolução (AFONSO et al., 2013). 


\section{Metodologia}

A análise foi realizada conforme pesquisa desenvolvida por Afonso et al. (2013, p. 511), denominado BrazilianSmartCitiesMaturityModel(Br-SCMM) - Modelo Brasileiro de Maturidade para Cidades Inteligentes, mensurando o quão inteligente uma cidade pode se tornar, tendo como base indicadores de Água, Saúde e Educação disponíveis em domínios públicos (AFONSO at al., 2013).

Os dados foram coletados do Sistema de Indicadores de Desenvolvimento Municipal Sustentável (SIDMS), realizado pela Federação Catarinense de Municípios (FECAM, 2014). Segundo Réus (2012, p. 23) o SIDMS tem por finalidade suprir a falta de informações de forma útil e consolidada sobre o desenvolvimento dos municípios catarinenses sob as perspectivas da sustentabilidade. Os índices são disponibilizados em uma plataforma online ${ }^{2}$, disponível para gestores públicos, pesquisadores e cidadãos sob a forma de ranking de acordo com os aspectos analisados. A Figura 1 ilustra os dados online na plataforma.

Figura 1 - Dados do Sistema de Indicadores de Desenvolvimento Municipal Sustentável.

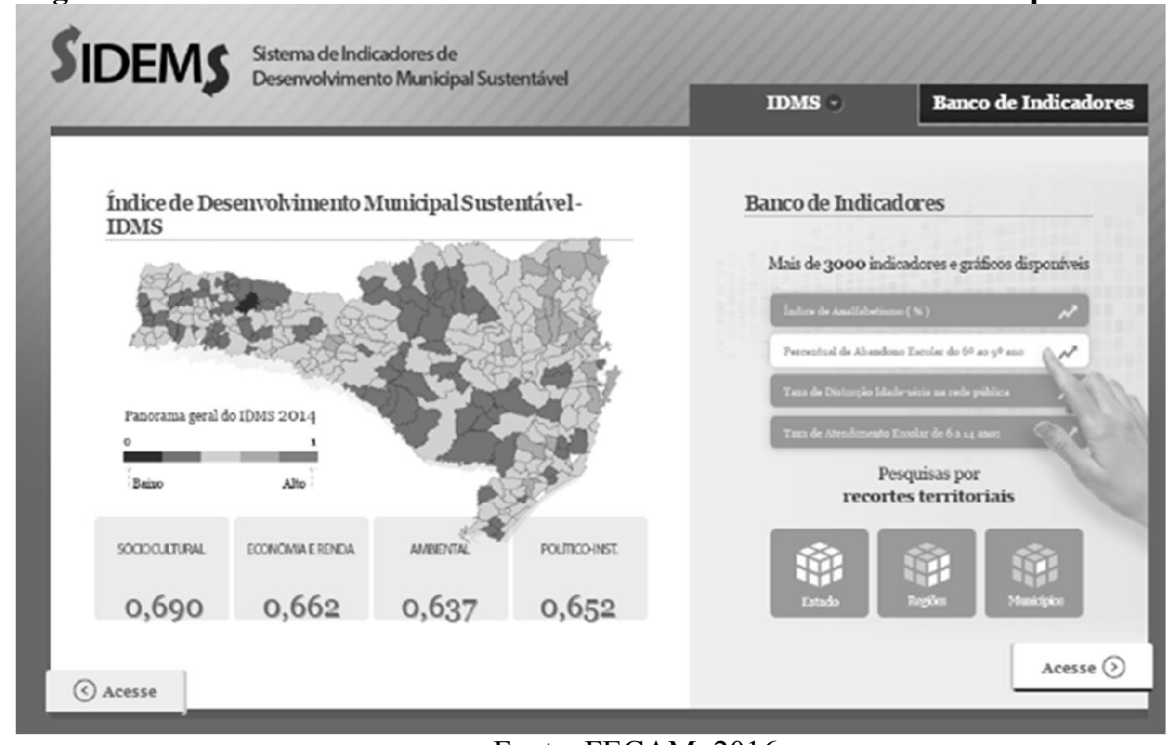

Fonte: FECAM, 2016

Os índices disponibilizados pelo SIDMS são apresentados em uma escala que vai de 0 a 1 , estratificados em cinco níveis de classificação onde, quanto mais próximo de 1 , melhor o desempenho, conforme demonstra a Tabela 1.

Tabela 1 - Níveis de classificação do Sistema de Indicadores de Desenvolvimento Municipal Sustentável.

\begin{tabular}{lc}
\hline \multicolumn{1}{c}{ Valor do IDMS } & Classificação \\
\hline Maior ou igual a 0,875 & ALTO \\
Maior ou igual a 0,750 e menor do que 0,875 & MÉDIO ALTO \\
Maior ou igual a 0,625 e menor do que 0,750 & MÉDIO \\
Maior ou igual a 0,500 e menor do que 0,625 & MÉDIO BAIXO \\
Menor do 0,500 & BAIXO \\
\hline
\end{tabular}

Fonte: FECAM (2014).

\footnotetext{
${ }^{2}$ Sistema de Indicadores de Desenvolvimento Municipal Sustentável (SIDMS). Disponível em: $<$ http://indicadores.fecam.org.br/>.
} 
Especificamente considerando os indicadores tratados por Afonso et al (2013), a FECAM apresentam dados para Educação, Saúde e Água, assim como ilustra o Quadro 2.

\begin{tabular}{|c|c|}
\hline Indicador & Descrição \\
\hline Educação & $\begin{array}{l}\text { No SIDMS são dois indicadores para a composição do desempenho } \\
\text { dentro da dimensão educacional. O primeiro mede sua qualidade, } \\
\text { através do levantamento da taxa de analfabetismo, as estatísticas de } \\
\text { evasão na educação básica, além das notas obtidas no Índice de } \\
\text { Desenvolvimento da Educação Básica (IDEB); enquanto o segundo } \\
\text { mede o percentual de cobertura do atendimento à população em idade } \\
\text { escolar. (FECAM, 2014). }\end{array}$ \\
\hline Saúde & $\begin{array}{l}\text { No SIDMS, o índice de Saúde é composto por indicadores de } \\
\text { mortalidade, fatores de risco cobertura de atenção básica à saúde, } \\
\text { incidência de doenças crônicas sobre a população e os recursos } \\
\text { disponíveis para os serviços (FECAM, 2014). A origem das } \\
\text { informações é do banco de dados da Secretaria de Estado da Saúde de } \\
\text { Santa Catarina. }\end{array}$ \\
\hline Água & $\begin{array}{l}\text { Para a análise, conforme indicações do SIDMS, foram reunidos dados } \\
\text { do percentual de domicílios atendidos por rede pública de } \\
\text { abastecimento de água, por meio dos indicadores da FECAM - } \\
\text { SIDMS. }\end{array}$ \\
\hline
\end{tabular}

Fonte: Elaborado pelos autores.

Desta forma, o artigo reuniu, para os municípios estudados, os índices nos domínios de Educação, Saúde e Água, calculando a média dos três domínios para cada uma das cidades. A classificação dos resultados obtidos será classificada conforme metodologia do SIDMS, nos parâmetros ilustrados no na Tabela 1 .

\section{A escolha das cidades}

O estado de Santa Catarina vem se destacando no cenário brasileiro pelos altos índices de desenvolvimento humano, considerando que Florianópolis possui o melhor desempenho dentre as capitais brasileiras (PNUD ONU, 2013). Além disso, Santa Catarina ocupa a terceira posição entre os estados se observada a média de seus municípios deste mesmo índice. Na atualidade o estado é responsável por aproximadamente 4\% do Produto Interno Bruto do país (FEPESE, 2016).

Para realização deste estudo, foram analisados os dados de seis municípios catarinenses com mais de 100.000 habitantes que obtiveram os melhores desempenhos no ranking de desenvolvimento sustentável promovido pela FECAM, sendo estes: Joinville,Florianópolis, Blumenau, Itajaí, Jaraguá do Sul e Balneário Camboriú ${ }^{3}$, assim como ilustra a Figura 2.

Figura 2 - Municípios catarinenses analisados no estudo.

\footnotetext{
${ }^{3}$ A apresentação dos municípios está conforme a ordem demográfica decrescente, segundo informações do Instituto Brasileiro de Geografia e Estatística.
} 


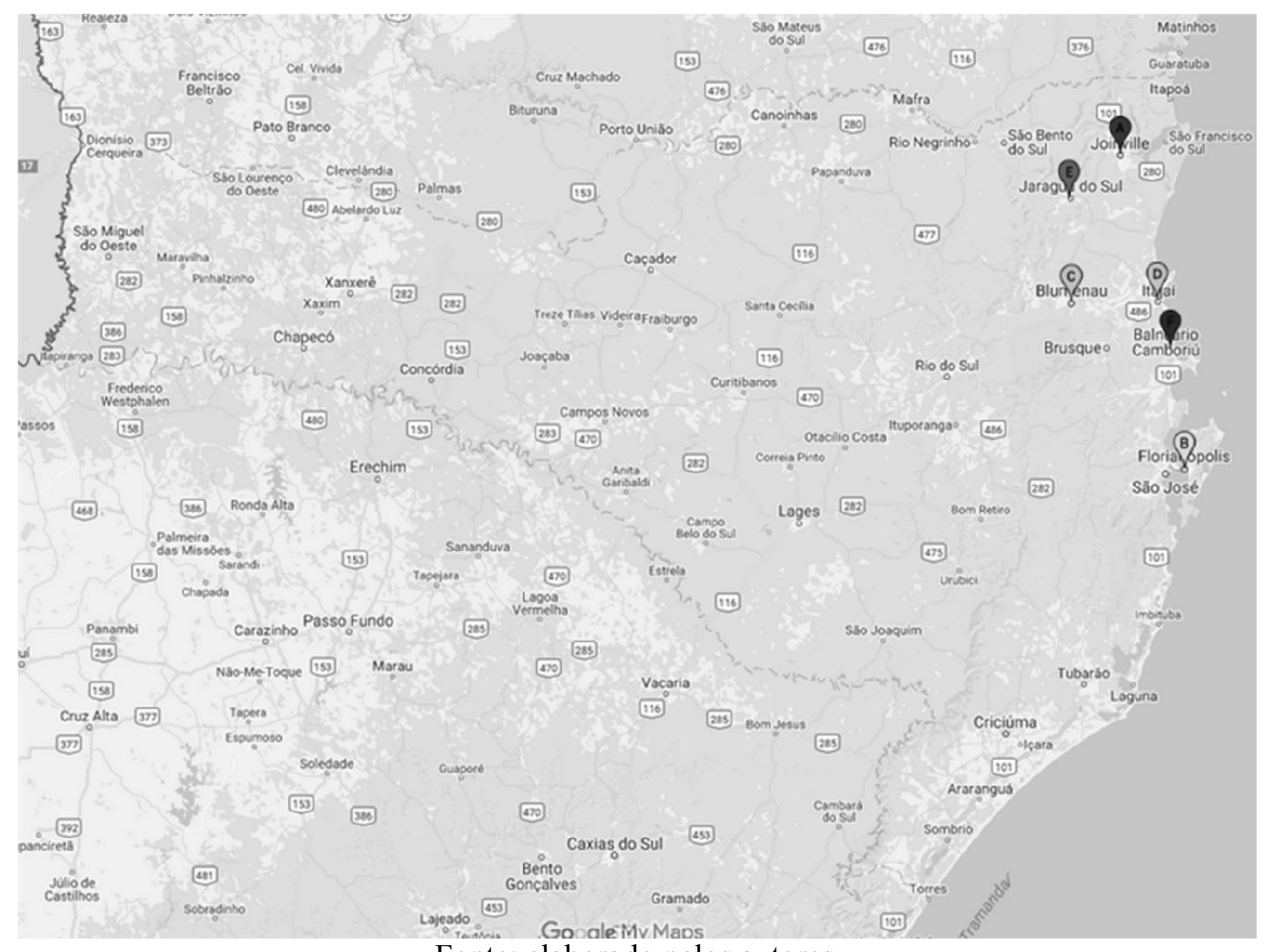

Fonte: elaborado pelos autores

\section{Resultados}

A partir do modelo de Afonso et al. (2013), foram levantados os índices nos domínios de Educação, Saúde e Água para os municípios abordado neste estudo. Foram calculadas as médias simples para cada cidade considerando os três domínios, além da média de cada um dos domínios considerando o grupo de todos os municípios, conforme mostra a Tabela 2.

Tabela 2 - Indicadores de desempenho obtido pelos municípios conforme SIDMS

\begin{tabular}{lcccc}
\hline Município & Educação & Saúde & Água & Média \\
\hline Joinville & 0,807 & 0,661 & 0,955 & 0,808 \\
Florianópolis & 0,732 & 0,773 & 0,936 & 0,814 \\
Blumenau & 0,803 & 0,778 & 0,931 & 0,837 \\
Itajaí & 0,804 & 0,691 & 0,950 & 0,815 \\
Jaraguá do Sul & 0,904 & 0,579 & 0,887 & 0,790 \\
Balneário. Camboriú & 0,791 & 0,648 & 0,963 & 0,801 \\
\hline Média & 0,807 & 0,688 & 0,937 & \\
\hline
\end{tabular}

Fonte: FECAM, 2014

Tomando os resultados para criação de um ranking de capacidade de transformação em cidades inteligentes, Blumenau alcançou o melhor desempenho $(0,837)$, seguido por Itajaí $(0,815)$, Florianópolis $(0,814)$, Joinville $(0,808)$, Balneário Camboriú $(0,801)$ e Jaraguá do Sul $(0,790)$. 
Ao realizar uma análise das médias, Blumenau é tida como a cidade com a maior capacidade de se tornar uma cidade inteligente, uma vez que apresentou a melhor média para os três domínios. Jaraguá do Sul ocupa a última posição não estando, porém, a uma realidade muito distante das demais cidades. Nota-se uma leve tendência de queda nos índices no primeiro nível, de forma proporcional ao número de habitantes de cada município. Considerando os níveis de classificação de desempenho sugeridos pelo SIDMS (FECAM, 2014), constatou-se que, na média, nenhum município alcançou o patamar máximo. Entretanto, todos os municípios se encontram no nível "médio alto".

Apesar de ocupar a última posição no ranking de capacidade de transformação em cidade inteligente, a Educação é um domínio de destaque em Jaraguá do Sul, justificados pela alta nota obtida no IDEB em 2011 para séries iniciais, combinado às baixas taxas de evasão escolar neste mesmo nível (FECAM, 2014).

Em contraste, a capital Florianópolis atingiu o nível considerado médio, proveniente do baixo desempenho no IDEB para os anos finais do ensino fundamental, bem como o abandono no ensino médio e a distorção "idade-série" na rede pública (FECAM, 2014). No sitio eletrônico da Prefeitura Municipal de Florianópolis foram encontrados projetos desenvolvidos pela Secretaria Municipal de Educação como forma de aprimorar os índices do município para o domínio de Educação. Os projetos visam o estímulo à leitura na idade escolar, alem da promoção à cultura, esporte e lazer. A gestão da educação no município está baseada no trabalho conjunto dos conselhos municipais de Alimentação Escolar, Educação e para o Fundo de Desenvolvimento da Educação Básica - FUNDEB (SECRETARIA MUNICIPAL DE EDUCAÇÃO DE FLORIANÓPOLIS, 2016). A Secretaria aponta ainda que com programas de implantação da educação em tempo integral, foram reduzidos os índices de evasão escolar e repetência entre os anos de 2012 e 2014.

Na saúde, todas as cidades atingiram nível médio, tendo a maioria alcançado médio ou médio baixo, com exceção de Florianópolis e Blumenau onde o desempenho é considerado médio alto. Jaraguá do Sul conta atualmente com a menor cobertura de serviços básicos em saúde familiar e saúde bucal, apresentando também baixos índices no número de consultas realizadas pelo Sistema Único de Saúde, por pessoa (FECAM, 2014).Conforme relatório de programação anual publicado pela Secretaria Municipal de Saúde de Jaraguá do Sul, um dos objetivos é ampliar a cobertura de atendimento em saúde familiar. A ação pretende implantar novas equipes de atendimento familiar, a partir de recursos da arrecadação municipal (SECRETARIA MUNICIPAL DE SAÚDE DE JARAGUÁ DO SUL, 2015). A Programação do município almeja ainda a contratação de novos profissionais em saúde especializados para ampliar a oferta de serviços em atenção especial.

Joinville e Balneário Camboriú apresentam baixos índices de cobertura pela saúde pública, considerando para tanto a população atendida por equipes de saúde bucal e a cobertura estratégica em programas de saúde familiar. Itajaí tem baixa cobertura de atendimento odontológico e de consultas através do Sistema Único de Saúde (FECAM, 2014).

No domínio de Água todos obtiveram índices considerados altos, com número médio de domicílios atendidos por rede de abastecimento de água superior a 93\%. Diferente da média estadual, onde apenas pouco mais de $60 \%$ dos domicílios possuem abastecimento de água em rede, o conjunto de municípios com maior capacidade de transformação em cidades inteligentes possui grande cobertura. O fato é justificado pela proporção de pessoas viventes em áreas urbanas nestas cidades, que supera 95\% (IBGE, 2010).

\footnotetext{
${ }^{4}$ A Taxa de Distorção Idade-série representa a quantidade de estudantes que estão cursando séries divergentes às consideradas para a sua idade.
} 


\title{
7. Considerações finais
}

O artigo buscou realizar a discussão entre os índices alcançados por seis municípios de Santa Catarina em três domínios considerados no Br-SCMM - Modelo Brasileiro de Maturidade para Cidade Inteligentes. Foram calculadas as médias para cada um, de forma a promover um ranking de capacidade de transformação em Cidade Inteligente, onde Blumenau ocupa a primeira posição. Em seguida, foram evidenciados os indicadores com maior relevância nos resultados, bem como a investigação de políticas públicas para o fortalecimento dos domínios com resultados mais desfavoráveis.

O Modelo de Maturidade encontra-se ainda em fase de desenvolvimento, não sendo possível a sua utilização em todos os níveis, pela inexistência de aplicação real nas cidades brasileiras. Apesar do fácil acesso aos dados para realização deste estudo especificamente, nem todos os municípios brasileiros possuem dados fiéis à realidade quanto as suas atuações nos determinados domínios.

Quanto à utilização dos domínios para mensuração da maturidade, enquanto o modelo europeu para mensuração dos níveis de inteligência das cidades utiliza de características como: economia, pessoas, governança, mobilidade, meio ambiente e estilo de vida, o modelo brasileiro resume-se apenas a índices que não são capazes de atingir todos os aspectos que compõem uma cidade. Para um levantamento mais completo, além dos domínios de educação, saúde e água, seria relevante a consideração de variáveis como segurança, tecnologia e economia. Além disso, o domínio de educação deve ser aprofundado, com informações não apenas da educação básica, mas também do ensino superior, considerando que as universidades são importantes intermediadores de conhecimentos e inovação.

A partir do estudo sugere-se o aprimoramento do modelo com a inclusão de novos domínios capazes de traduzir de maneira mais completa a maturidade das cidades inteligentes. Além disso, é importante a aplicação efetiva do modelo na administração do município, como forma de possibilitar a comparação de evolução dos índices.

\section{Referências}

\section{AFONSO, R. A. et al. Br-SCMM: Modelo Brasileiro de Maturidade para Cidades Inteligentes}

\author{
Atlas do Desenvolvimento Humano no Brasil. Programa das Nações Unidas para o \\ Desenvolvimento Humano. 2013. Disponível em: \\ $<$ http://www.atlasbrasil.org.br/2013/pt/ranking $>$
}

Caminho para as smartcities: da gestão tradicional para a cidade inteligente / Maurício Bouskela, Márcia Casseb, Silvia Bassi, Cristina De Luca y Marcelo Facchina. p. cm. - (Monografia do BID ; 454)

Censo Demográfico. Instituto Brasileiro de Geografia e Estatística - IBGE. 2010. Disponível em: <http://censo2010.ibge.gov.br/>

ConnectedSmartCities. Cidades do Futuro do Brasil. 2016. Disponível em: $<$ http://www.connectedsmartcities.com.br/>

DEAKIN, M. ALLWINKLE, S. Urban regenneration and sustainable communities: the role networks, innovation and creativity in building successful partnerships. Journal of Urban Technology 14: 77-91. 
European Smart Cities.Vienna University of Technology.2016. Disponível em:<http://www.smart-cities.eu/>

HOLLANDS, R.G. Will the real smart city please stand up? Intelligent, progressive or entrepreneurial?City, 12 (3), pp. 303-320, 2008.

IESE Cities in Motion.IESE Business School.Universityof Navarra. 2014. Disponível em: $<$ http://www.ieseinsight.com/doc.aspx?id=1582>

Informações em Saúde - Programação Anual. Secretaria Municipal de Saúde de Jaraguá do Sul, 2015. Disponível em: $<$ http://www.jaraguadosul.sc.gov.br/downloads.php?id=15600>

KOMNINOS, N. Intelligent Cities: Innovation, Knowledge Systems and Digital Spaces. London: Routledge, 2002.

MALEK, A. J. Informative Global community development index of informative smart city.In proceedings of the $8^{\text {th }}$ WSEAS International Conference on Educations and Educational Technology.Genova, Italy, 17-19.

NEGRI, João Alberto; LEMOS, Mauro Borges. Avaliação das Políticas de Incentivo à P\&D e Inovação Tecnológica no Brasil. Mimeo - Nota Técnica do IPEA para o Conselho Nacional de Ciência e Tecnologia/MCT.

Portal da Economia de Santa Catarina. Fundação de Estudos e Pesquisas Socioeconômicas. 2016. Disponível em: < http://novosite.fepese.org.br/portaldeeconomia-sc/>

Prefeitura Municipal de Blumenau. 2016. Disponível em:http://www.blumenau.sc.gov.br/

Prefeitura Municipal de Balneário Camboriú. 2016. Disponível em:

$<\underline{\text { http://www.balneariocamboriu.sc.gov.br/index.cfm }>}$

Prefeitura Municipal de Florianópolis. 2016. Disponível em: $<\underline{\text { http://www.pmf.sc.gov.br/> }}$

Prefeitura Municipal de Itajaí. 2016. Disponível em: <http://www.itajai.sc.gov.br/>

Prefeitura Municipal de Jaraguá do Sul. 2016. Disponível em:

$<\underline{\text { http://www.jaraguadosul.sc.gov.br/> }}$

Prefeitura Municipal de Joinville. 2016. Disponível em: < $\underline{\text { https://www.joinville.sc.gov.br/ }>}$

Projetos da Secretaria Municipal de Educação. Secretaria Municipal de Educação de Florianópolis. 2016. Disponível em:

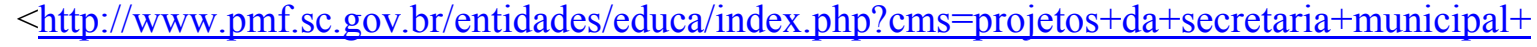
$\underline{\text { de}+ \text { educacao\&menu }=1>}$

RAMOS JUNIOR, H. S; GALIOTTO, S. Iniciativas Pontuais de Cidades Inteligentes no

Meio-oeste Catarinense: estudo do caso da cidade de Erval Velho, a Capital Catarinense da Reciclagem. Revista Democracia Digital e Governo Eletrônico (ISSN 2175-9391), nº 9, p. 4465, 2013.

Sistema de Indicadores de Desenvolvimento Municipal Sustentável. Federação Catarinense de Municípios - FECAM. 2014. Disponível em: $<\underline{\text { http://indicadores.fecam.org.br/> }}$

United Nations, Department of Economic and Social Affairs, Population Division (2014). World Urbanization Prospects: The 2014 Revision, CD-ROM Edition.

SOUZA, R. A. CARVALHO, A. M. Programa de Saúde da Família e qualidade de vida: um olhar da psicologia. Estudos de Psicologia 2003, 8(3), 515-523. 